\title{
Preocupación ambiental y formación ambiental en estudiantes universitarios
}

\author{
Edith Meryluz Claros Guerrero \\ eclaros@unjfsc.edu.pe \\ meryluzclaros@gmail.com
}

Cristian Iván Escurra Estrada

cescurra@unjfsc.edu.pe

Universidad Nacional José Faustino Sánchez Carrión

Huacho - Perú

\section{RESUMEN}

La investigación se basa en la tesis doctoral ambientalización de la Universidad Nacional José Faustino Sánchez Carrión, que evalúa la dimensión ambiental desde un punto de vista integral, abordándose el problema de la percepción que tiene el estudiante universitario de su formación profesional en materia ambiental (plan curricular, metodología docente, contenido) al margen de su formación profesional en relación con la autovaloración de sus actitudes pro ambientalistas (preocupación ambiental). A través del muestreo probabilístico estratificado se determinó el tamaño de la muestra de cada carrera profesional seleccionada intencionalmente para cumplir con el objetivo de la investigación. Se aplicó un cuestionario basado en la propuesta de Melania Coya, a los estudiantes del III al V Semestre, de 10 escuelas profesionales, quienes valuaron su formación en materia ambiental, y la escala de preocupación ambiental. Los estudiantes encuestados obtuvieron una puntuación de preocupación ambiental de 44.02 puntos de 65, calificándose en el grado medio, con nivel regular de formación en materia ambiental. No hay correlación significativa entre formación ambiental y preocupación ambiental, a excepción de las carreras profesional con perfil profesional afín a la temática ambiental, concluyéndose que la preocupación ambiental de los estudiantes se ve influenciado por el entorno cultural y social.

Palabras clave: formación ambiental; preocupación ambiental; metodología, perfil profesional 


\title{
Environmental concern and environmental training in university students
}

\begin{abstract}
The research is based on the doctoral thesis environmentalization of the José Faustino Sánchez Carrión National University, which assesses the environmental dimension from an integral point of view, addressing the problem of the perception that university students have of their professional training in environmental matters (plan curriculum, proffesor methodology, content) aside from their professional training in relation to self-assessment of their pro-environmental attitudes (environmental concern). Through stratified probability sampling, the sample size of each professional career selected intentionally to meet the objective of the research was determined. A questionnaire based on the proposal of Melania Coya was applied to students from the III to the V Semester of 10 professional career, who valued their training in environmental matters, and the scale of environmental concern. The surveyed students obtained a score of environmental concern of 44.02 points out of 65 , qualifying in the medium grade, with a regular level of training in environmental matters. There is no significant correlation between environmental training and environmental concern, with the exception of professional careers with a professional profile related to environmental issues, concluding that the environmental concern of students is influenced by the cultural and social environment.
\end{abstract}

Keywords: environmental training; environmental concern; methodology, professional profile

Artículo recibido: 15 noviembre. 2021 Aceptado para publicación: 10 diciembre 2021 Correspondencia: meryluzclaros@gmail.com Conflictos de Interés: Ninguna que declarar 


\section{INTRODUCCIÓN}

La problemática ambiental, en si, es una temática muy compleja de abordar, y que se acentúa más aun en las últimas décadas, evidenciándose un incremento considerable de consecuencias en diferentes factores, como el factor físico, biótico y social, que van afectando las actividades cotidianas y que a pesar de las acciones que se realiza a nivel global para lograr la sensibilización del cuidado y respeto al medio ambiente, a través de la emisión de normativas pro ambientalistas que buscan la sostenibilidad ambiental, aun así pareciera que el modelo de desarrollo social y económico aparentemente no está logrando conseguir en su totalidad el equilibrio natural y el bienestar de los ecosistemas, por ello, es necesario, que la idea de sostenibilidad esté inmerso y forme parte de la cultura del ser humano, una alternativa es incentivar la educación ambiental, formal e informal, mediante la interacción de los diversos actores que conforman la sociedad entre ellos los ciudadanos, grupos organizados de la sociedad, gubernamentales, empresariales y sobre todo instituciones educativas, especialmente las instituciones de educación superior, para subsanar y mitigar los efectos que conlleva la crisis ambiental. Otro concepto inmerso en la problemática ambiental es la sustentabilidad, y tal como lo menciona (García, D \& Priotto, 2009) ello implica asumir la responsabilidad de mejorar las condiciones actuales de vida de todas las personas, de las demás formas de vida y de los sistemas naturales. La institución superior universitaria, ámbito en el cual se forman los profesionales con diversos perfiles ocupacionales, quienes debe ser conscientes de la realidad social, ambiental y ser partícipes activos de la transformación de la sociedad con responsabilidad ambiental, tal como lo menciona Mora (2012) quien considera que incluir la dimensión ambiental en la Educación Superior es imperativo, y ello se debe dar a través de la aplicación de modelos centrados en el desarrollo sostenible, por su parte (Silva, 2007) considera que al incluir la dimensión ambiental en el sistema educativo debe conducir hacia las nuevas formas de relación entre los seres humanos y la naturaleza, de éstos entre sí y con el resto de sociedad, ello conllevará a lograr la formación de la conciencia ambiental como necesidad inmediata en la preparación de los futuros graduados universitarios. Noguera(2007)

Según Cárdenas (2013a), la responsabilidad ambiental universitaria es la acción de la universidad puesta en práctica en principios y valores que contribuye en la formación de profesionales y ciudadanos con conciencia, compromiso y participación proactiva en la 
solución de los problemas ambientales, mediante el ejercicio de sus funciones sustantivas de formación, investigación, extensión y gestión, el autor considera además que esta institución debe incorporar la dimensión ambiental a su proyecto institucional, educativo y laboral

Si analizamos la normatividad nacional, en el art. $127^{\circ}$ de la política nacional de educación ambiental de nuestro país, menciona que: "la educación ambiental se convierte en un proceso educativo integral que se da en toda la vida del individuo, que busca generar en éste los conocimientos, actitudes, valores y prácticas, necesarios para desarrollar sus actividades en forma ambientalmente adecuada, con miras a contribuir al desarrollo sostenible del país", en ese sentido el Perú a través del Ministerio del Ambiente (MINAM) mediante la Red Ambiental Interuniversitaria - Interuniversia Perú (RAI Perú), ha iniciado un proceso desde el año 2012 (Cárdenas, 2018c); y es concordante con lo previsto en el Plan Bicentenario - El Perú hacia el 2021

Una educación ambiental óptima con valores y actitudes ambientalmente responsables, sería considerar la dimensión ambiental como parte de la formación profesional, adaptando el currículo universitario que influya en un cambio de conducta de toda la comunidad universitaria, interiorizándose el respeto al medio ambiente y los recursos naturales.

Según la Ley Universitaria (Ley $\mathrm{N}^{\circ} 30220,2014$ ): "La universidad es una comunidad académica orientada a la investigación y a la docencia, que brinda una formación humanista, científica y tecnológica con una clara conciencia de nuestro país como realidad multicultural". Y entre sus fines entre otros es de "promover el desarrollo humano y sostenible, servir a la comunidad y buscar el desarrollo integral”,

Este documento se basa en la tesis doctoral Ambientalización de la Universidad Nacional José Faustino Sánchez Carrión (Claros, 2020) donde se realizó un diagnóstico de las condiciones de ambientalización en la que se encuentra la institución educativa, siendo motivo de análisis el grado de preocupación ambiental del estudiante Universitario que permite conocer la percepción del estudiante frente a la problemática ambiental, y el nivel de formación en materia ambiental que bajo la percepción del estudiante viene recibiendo como parte de su formación profesional, al margen de su perfil profesional. 


\section{ESTRATEGIAS METODOLÓGICAS O MATERIALES Y MÉTODOS}

El diseño de la investigación tuvo un enfoque cuantitativo, correlacional de corte transversal, la población estuvo compuesta por estudiantes matriculados en el Semestre 2019 - I, distribuido en 13 Facultades con 34 Carreras profesionales, algunas afines a la temática ambiental, y otras que "no son afines", para hacer la diferencia se analiza el plan curricular de las carreras profesionales, que según normatividad de la institución, se divide en tres áreas curriculares: Área de estudios generales o formación básica, área de estudios específicos o formación profesional básica y el área de especialidad o formación profesional especializada, siendo la formación básica, donde se identifica la competencia en ciencia, tecnología y relación con el medio físico, cursándose en los primeros cuatro ciclos.

En base a las recomendaciones de Fox (1987, 2a: 373) citado por (Coya, 2001) que menciona que para conseguir deliberadamente la representatividad de la muestra deben cumplirse tres condiciones: a)conocer las características (variables) que están relacionadas con el problema que se estudia; b) tener la capacidad para medir esas características; c) poseer datos de la población sobre las características para utilizarlos como base de comparación y el incumplimiento de una de estas condiciones debería justificar el uso de otro tipo de método, se restringe la población universitaria a los ciclos de III a VI Ciclo, descartándose los primeros ciclos asumiendo que no tienen suficiente información para juzgar la formación profesional y los estudiantes de ciclos superiores, para lograr homogeneidad, pues no todos cursan en un plan por competencias, se elige solo una carrera profesional de aquellas que tienen planes curriculares afines. Quedando la población en 3183 estudiantes, distribuidos en 10 carreras profesionales. Mediante el muestreo no probabilístico, con criterio condicionado se hicieron las exclusiones que requiere la investigación, encuestándose a 278 estudiantes.

A los estudiantes encuestados se les aplicó un cuestionario adaptado a la realidad y características de la región, para conocer la formación ambiental, y para el grado de preocupación ambiental, se utilizó la Escala de Preocupación ambiental, propuesto por Coya (2001), tomando como base la Environmental Concern Scale (Escala de Preocupación Ambiental, EPA) diseñada por Weigel y Weigel (1978), adaptados por Holahan (1991) o Amerigo y Gonzales (1996). Se identificaron cuatro dimensiones para la evaluación de la preocupación ambiental: Control no personal sobre el medio ambiente 
que evalúa si el estudiante asume el control de la contaminación y presenta la solución, Esfuerzo personal por la conservación del medio ambiente, si el estudiante está dispuesto en asumir algún coste personal, prevención y control de la contaminación, el estudiante espera que las autoridades soluciones o plantean alternativas de solución, y actitud irresponsable hacia la contaminación se evalúa si el estudiante valora el real importancia de la contaminación y se hace responsable de sus acciones. En el análisis documental se hizo la revisión de los planes curriculares, observándose la cantidad de cursos por áreas temáticas, registrados en la página Web de la institución.

\section{RESULTADOS Y DISCUSIÓN}

Para una muestra de 278 estudiantes, con edad promedio de 20 años, conformada por estudiantes de las carreras no afines al área ambiental: Estadística e Informática (4\%), Economía y Finanzas $(15,1 \%)$, Ingeniería Química (10.1\%), Ingeniería Electrónica $(10.8 \%)$, Sociología $(9 \%)$, Enfermería $(10.1 \%)$, mientras que las Escuelas afines a la materia ambiental son Ingeniería Zootécnica (7,9\%), Biología con mención en Biotecnología (6.1\%), Ingeniería Ambiental (14,4\%), Ingeniería Agronómica (12,6\%).

Análisis Descriptivo: Preocupación ambiental: De los 5 ítems con actitudes negativas, 8 con actitudes positivas, de la Escala de Preocupación ambiental, distribuidos en cuatro dimensiones que evalúen las actitudes de los estudiantes obteniéndose en un rango de 13 - 65 y promedio potencial de 44,02, se ubica en el grado de preocupación ambiental medio.

\section{Tabla 1}

Dimensiones de la Escala de Preocupación ambiental

\begin{tabular}{lccc}
\hline Dimensiones EPA & $\begin{array}{c}\text { Rango } \\
\text { potencial }\end{array}$ & $\begin{array}{c}\text { Promedio } \\
\text { potencial }\end{array}$ & Conclusión \\
\hline Control no personal sobre el medio ambiente & $03-15$ & 8.953 & Medio (-) \\
$\begin{array}{l}\text { Esfuerzo personal por la conservación del } \\
\text { medio ambiente }\end{array}$ & $04-20$ & 14.809 & Alto (+) \\
$\begin{array}{l}\text { Prevención y control de la contaminación } \\
\text { actitud irresponsable hacia la contaminación }\end{array}$ & $04-20$ & 15.845 & $\begin{array}{l}\text { Alto (+) } \\
\text { Bajo (-) }\end{array}$ \\
\hline
\end{tabular}

Nota: (-) Actitud no favorable (+) Actitud favorable

Si analizamos los resultados por Carrera o Escuela profesional, se obtiene que Ingeniería

Ambiental, tiene resultados positivos y se explica por su perfil profesional, según las 
dimensiones consideradas en la investigación mientras que las Escuelas con poca o ninguna formación en materia ambiental tienen promedios potenciales bajos.

En relación a la Formación Ambiental; En opinión de los estudiantes encuestados de la Universidad Nacional José Faustino Sánchez Carrión, para el 65,47\% es Regular, considera Buena el $19.06 \%$ y no ha tenido ninguna formación en materia ambiental el $15.47 \%$, específicamente se está valorando la formación ambiental en el contenido teórico, el mayor porcentaje consideran que en los cursos que han llevado, incentivan parcialmente a rescatar valores, la identidad nacional, y contenido con temática ambiental.

Referente al Plan Curricular, en base a la información documental, las escuelas tienen cursos con contenidos en temática ambiental como parte de su plan curricular de más a menos: Ingeniería Ambiental, Ingeniería Agronómica, Biología con mención en Biotecnología, e Ingeniería Zootécnica; y las escuelas que no tienen cursos con temática ambiental son: Ingeniería Electrónica, Ingeniería Química, Estadística e Informática, Economía y Finanzas; coincidiendo con lo obtenido por (Venegas \& Quijano, 2017) quienes concluyen Administración y contabilidad, no contienen asignaturas relacionadas con el medio ambiente, mientras tanto en la escuela de Enfermería sus contenidos temáticos están relacionadas al aspecto humanístico.

Sobre la Calidad en Formación ambiental recibida, los estudiantes que tienen Opinión favorable son los de la Escuela de Ingeniería Ambiental; para los de Ingeniería Zootécnica, Enfermería, Biología con mención en Biotecnología, Estadística e Informática, consideran que la formación les permitirá conocer, entender, enfrentar y actuar en temática ambiental en más del $50 \%$ de los casos. Donde existe cierta negatividad en éste aspecto, es en los estudiantes de Sociología, Economía y Finanzas (mayor cantidad) e Ingeniería Química en menor porcentaje.

En relación a la metodología docente referido a temática ambiental: Los estudiantes de Ingeniería Ambiental, Ingeniería Agronómica, Ingeniería Zootécnica y Biología con mención en Biotecnología, consideran que los docentes han desarrollado dentro de los contenidos y metodología la temática ambiental en mayor proporción; en Economía y Finanzas, consideran que los docentes, no desarrollan nada referente a la temática ambiental como parte de la metodología de los cursos, resultado coincidente con lo 
obtenido por (Padilla, 2016), quien en su investigación obtuvo que la capacitación de los docentes aun es incipiente, lo que contrasta con la actitud estudiantil que es positiva.

Análisis correlacional: Para determinar si el grado de preocupación ambiental se relaciona con la formación ambiental de los estudiantes de la Institución, en el análisis exploratorio de datos, para determinar si la muestra se ajusta a una distribución normal, hacemos uso de Kolgomorov - Smirnov (corrección de la significancia de lilliefors), se obtiene para formación ambiental con un valor de 0.070 y $\mathrm{p}=0,002<0,05$ se rechaza la hipótesis nula, concluyéndose que no existe normalidad en los datos; y para la variable preocupación ambiental con un valor de 0.062 y p=0.011<0.05, se concluye que los datos no son normales. Considerando que son datos ordinales y no paramétricos en ambas variables, hacemos uso del coeficiente de correlación rho de Spearman, y para $\mathrm{r}=0.081$ con $\mathrm{p}=0.179>0.05$, se concluye que no existe correlación significativa entre las variables. Ante la pregunta: Si el nivel de formación ambiental que está recibiendo de parte de la Universidad, se correlaciona con la medida que los estudiantes consideran la importancia del medio ambiente, se obtiene $r=0.109$ y $\mathrm{p}=0.071>0.05$, lo que nos indica que a pesar que si es necesario tener como base una formación profesional en temática ambiental, para conocer, interpretar, y promover acciones en pro del medio ambiente, eso no implica que no se considere y valore la importancia del medio ambiente(62,59\%), y de manera similar por cada escuela.

\section{- En relación a la correlación de las dimensiones de preocupación ambiental:}

El control no personal sobre el medio ambiente está correlacionada significativamente con el esfuerzo personal que el estudiante está dispuesto realizar por la conservación del medio ambiente $\left(\mathrm{r}=0.135^{*}, \mathrm{p}=0.025<0.05\right)$; a la vez se correlaciona significativamente con la actitud irresponsable hacia la contaminación, de manera directa $\left(\mathrm{r}=, 435^{* *}\right.$ $\mathrm{p}=, 000<0,01$ ) (ambas actitudes negativas).

Se observa también que el estudiante es consciente que tiene que formar parte activa para frenar la contaminación y buscar la protección del medio ambiente, lo que se demuestra estadísticamente $(\mathrm{r}=, 571 * * ; \mathrm{p}=, 0000<0.01)$ que existe alta correlación del esfuerzo personal por la conservación del medio ambiente y lograr la prevención y control de la contaminación, con una relación directa y positiva.

Se obtiene que existe correlación significativa entre la prevención y control de la contaminación con actitud irresponsable hacia la contaminación $(r=-, 350 ; p=, 000<0,01)$, 
cuya relación es indirecta, es decir que mientras la actitud irresponsable disminuye, se incrementará la búsqueda de prevención y control de la contaminación.

\section{- En relación a la correlación de las dimensiones de Formación Ambiental:}

Respecto a la formación humanística y pro ambientalista en los contenidos de los cursos se correlaciona con el número de cursos del plan curricular( $\mathrm{r}=, 415, \mathrm{p}=, 000<, 01)$, de manera directa y positiva, lo que implica que a mayor cantidad de cursos con temática ambiental, mayor será la formación humanística; y a la vez se correlaciona significativamente con la calidad en la formación ambiental $(r=, 302 ; p=, 000<, 01)$, y con la metodología que utiliza el docente en el desarrollo de los contenidos $\left(\mathrm{r}=, 411^{* *}\right.$; $\mathrm{p}=, 000<, 01)$.

La cantidad de cursos que forman parte del plan curricular en materia ambiental, se correlaciona significativamente $\left(\mathrm{r}=, 547^{* *}, \mathrm{p}=, 000<, 01\right)$ con la calidad de la formación en temática ambiental, y con la metodología docente (con un plan curricular con mayores cursos relacionados a la temática ambiental $(\mathrm{r}=, 632 * *, \mathrm{p}=, 000<, 01)$; y por su parte la calidad de la formación universitaria esta correlacionada significativamente con la metodología que utiliza el docente en materia ambiental $\left(\mathrm{r}=, 507^{* *}, \mathrm{p}=, 000<, 01\right)$.

- Correlación entre las dimensiones de preocupación ambiental y dimensiones de la formación ambiental:

Se observa alta correlación significativa entre la formación humanística y pro ambientalista en los contenidos de los cursos, con el control no personal sobre el medio ambiente, de manera directa y positiva ( $\mathrm{r}=, 192, \mathrm{p}=, 001<0.01$ ), y con la metodología que el docente utilice en el aula en materia ambiental $(\mathrm{r}=, 150 ; \mathrm{p}=, 012<, 05)$; lo que implica que mientras se incentive a la formación en valores éticos, análisis crítico de la realidad, y se utilice por parte del docente, metodologías que incluya la dimensión ambiental en su cátedra con ejemplos prácticos, los estudiantes tendrá mayor incentivo para participar activamente con sentido de responsabilidad y adoptaran posiciones que conlleven al control de la contaminación y conservación del medio ambiente.

La calidad de la formación universitaria en temática ambiental está correlacionada con el esfuerzo personal que el estudiante manifiesta, está, dispuesto a realizar por la conservación del medio ambiente $(\mathrm{r}=, 131 ; \mathrm{p}=, 030<0,05)$, con adoptar acciones $\mathrm{y}$ participar activamente en la prevención y control de la contaminación $(\mathrm{r}=, 231$; $\mathrm{p}=, 000<, 01)$. 
En relación a la correlación del nivel de formación ambiental, con el grado de preocupación ambiental de los estudiantes por escuela profesional, tiene correlación significativa a un nivel de significancia de 0,05 en la Escuela de Ingeniería Ambiental $\left(r=, 375^{*} ; \mathrm{p}=, 017<0,05\right)$, entre el nivel de Preocupación Ambiental y su formación en materia ambiental, con una relación positiva y existe correlación a un nivel de significancia de 0,01 en la Escuela de Sociología para $(r=, 549 * * ; p=, 005<0,01)$ entre ambas variables, mientras que las escuelas restantes, estadísticamente no hay correlación significativa entre ambas variables $(\mathrm{p}>0,05)$

Berenguer y Corraliza (2000) menciona que “cuando se evalúa la preocupación por el medio ambiente a nivel general se registran elevados niveles de preocupación ambiental, sin embargo esa evaluación no es suficiente para predecir otras creencias y valores pro ambientales", en ésta investigación se les preguntó a los estudiantes que se autoevalúen y respondan la importancia que ellos le dan al medio ambiente, y se obtuvo que para el $62,59 \%$ el medio ambiente es muy importante para ellos, y mediante la evaluación de la escala de preocupación ambiental(EPA) tiene una puntuación con promedio potencial de 44,02 puntos de 65 como máximo, que corresponde a un grado de preocupación ambiental media $(61,5 \%)$ con estudiantes que tiene un grado de preocupación alta $(35,97 \%)$.

"La preocupación ambiental ha sido considerada un parámetro descriptivo del nivel de concienciación ambiental, y una variable predictora del comportamiento ambiental" (Berenguer \& Corraliza, 2000), bajo esta premisa se observó que los estudiantes en su mayor porcentaje conocen la existencia de la contaminación en lagos, ríos, aire, pero consideran que la naturaleza a través de los procesos de purificación lo retornará a la normalidad (control no personal sobre el medio ambiente), sin embargo están dispuestos a realizar sacrificios personales ya sea económicos, de tiempo, u otras actividades para prevenir la extinción de animales y reducir el ritmo de la contaminación (esfuerzo personal por la conservación del medio ambiente); como parte de las acciones de prevención y control de la contaminación, así como exigir a las instancias gubernamentales su participacion directa para el control de la contaminación, pero además consideran que los colegios de educación secundaria deben ser los primeros en impartir educación sobre la conservación de los recursos naturales, ésta afirmación se relaciona con lo que expresan Amérigo y García, (2014): "las personas valoran su 
preocupación por los problemas ambientales en función de que estos tengan consecuencias dañinas para uno mismo, los demás seres humanos o la biosfera”.

El mayor porcentaje de estudiantes encuestados consideraron que la formación en materia ambiental recibida es Regular y concuerda con el análisis de los indicadores propuestos por Cárdenas (2014); quien considera que la incorporación de la perspectiva ambiental en la función formación de las universidades es de moderada a baja.

Las actitudes pro ambientalistas por parte de los estudiantes se ve influenciada por su perfil profesional, como la Escuela de Ingeniería Ambiental, cuya formación está correlacionada con el grado de preocupación ambiental, a diferencia de las otras escuelas analizadas en ésta investigación, lo que concuerda con (Casas \& Jaula, 2004) que considera que la universidad desde su tratamiento tradicional, es responsable de la fragmentación de los campos del conocimiento y de gran parte de los efectos negativos que ello ha provocado sobre el medio natural y social; pero también es de resaltar que la Escuela de Sociología que a pesar de no contar con un perfil profesional "ambientalizado", existe correlación en ambas variables, lo que se explica que la actitudes positivas en relación al medio ambiente, no sólo está condicionado a la formación académica o educación formal, si no en base al entorno, cultura y costumbres que son innatas en el ser humano, como lo menciona (Delgado, 2012) que la educación informal se refiere a los diversos aprendizajes logrados mediante las experiencias cotidianas en la casa, trabajo, calle, viajes, etc, al conversar, leer o utilizar medios masivos de comunicación y considera el autor que a ella se debe el bagaje de información, habilidades y actitudes que poseen las personas.

\section{CONCLUSIÓN O CONSIDERACIONES FINALES}

Las actitudes pro ambientalistas como la preocupación ambiental en los estudiantes universitarios no sólo se ve influenciada por su educación formal o formación profesional, sino que depende de los diversos aprendizajes que adquieren de las actividades de su vida cotidiana y del entorno cultural y social.

Asumir como política institucional de manera integral la política ambiental, que permitirá que los estudiantes y futuros profesionales identifiquen problemas ambientales que ocasionan la disminución de la calidad de vida, tendrán un enfoque interdisciplinario y serán actores activos en el desempeño de sus labores y actividades profesionales en pro de la sostenibilidad. 
Es importante, generar un plan de formación y actualización docente como política académica, con un enfoque interdisciplinario, con trabajo colaborativo que permita adoptar e interiorizar la cultura ambiental, mejorando el desempeño colectivo y ambientalmente responsable en la Universidad.

\section{LISTA DE REFERENCIAS}

Amérigo, M., \& García, J. (2014). Perspectiva Multidimensional de la Preocupación por el Medio Ambiente. Relación entre Dimensiones Actitudinales y comportamientos. PSico, 406-414.

Berenguer, J., \& Corraliza, J. A. (2000). Preocupación ambiental y comportamientos ecológicos. Psicothema, 325-329.

Cardenas, J. (2013). Guia para Universidades Ambientalmente Responsables. Responsabilidad Ambiental Universitaria: Compromiso y oportunidad. Red Ambiental Interuniversitaria - Interuniversia Perú. .

Cárdenas, J. (2014). Incorporación de la Perspectiva Ambiental en las Universidades Peruanas- Reporte sobre el compromiso Ambiental de las Universidades . RED AMBIENTAL INTERUNIVERSITARIA. INTERUNIVERSIA PERÚ, 1-34.

Cárdenas, J. (2018). Matriz de Indicadores de Incorporación de la dimensión ambiental en Universidades (Versión actualizada). Lima : Red Ambiental Interuniversitaria - Interuniversia Perú (RAI).

Casas, M., \& Jaula, A. (2004). Intervenciones ambientalizadoras en la Universidad de Pinar del Rio, Cuba. Red ACEs, 173-186.

Claros, E. (2020). Ambientalización de la Universidad Nacional José Faustino Sánchez. Carrión. Tesis doctoral, Universidad Nacional José Faustino Sánchez Carrión, Huacho. Perú.

Coya, M. (2001). La ambientalización de la Universidad Santiago de Compostela. Tesis doctoral, Universidad de Santiago de Compostela, España.

D.S. N054-2011-PCM. (23 de Junio de 2011). Plan Bicentenario: El Perú hacia el 2021. Diario Oficial El Peruano, Perú.

Delgado, K. (2012). Educación Ambiental. Experiencias y propuestas. Lima: San Marcos. 
García, D, \& Priotto, G. (2009). Educación Ambiental. Aportes políticos y pedagógicos en la construcción del campo de la Educación Ambiental. Buenos Aires : Secretaría de Ambiente y Desarrollo Sustentable de la Nación.

Hernández, R., Fernández, C., \& Baptista, M. (2014). Metodología de la Investigación. México: McGraw Hill /Interamerica Editores S.A de C.V.

Ley N³0220. (09 de Julio de 2014). Ley Universitaria. Perú: Diario Oficial El Peruano. Macedo, L. (2014). Política Ambiental de la Facultad de Ciencias de la Educación y Humanidades - UNAP (Tesis de Maestria ). Perú: Universidad Nacional del Altiplano.

Mora, W. M. (2012). AMBIENTALIZACIÓN CURRICULAR EN LA EDUCACIÓN SUPERIOR:UN ESTUDIO CUALITATIVO DE LAS IDEAS DEL PROFESORADO. Revista de Currículum y Formación de Profesorado [en linea] 2012, , 75-105. Recuperado el 2019

Noguera, A. (2007). Emergencias de un pensamiento ambiental alternativo en América Latina. Colombia : Universidad Nacional - IDEA.

Padilla, R. (2016). Ambientalización curricular, las actitudes hacia la educación ambiental y su relación con la calidad de vida de los estudiantes de la Facultad de Educación de la UNMSM. Lima, Perú: Universidad Nacional Mayor de San Marcos.

Silva, J. (2007). Introducción de la dimension ambiental en lo curricular en la enseñanza universitaria. Hojas de Sol en la Victoria Regia, 149-179. Colombia.

Venegas, L., \& Quijano, Y. (2017). Ambientalización Curricular: Mejora en Formación de futuros egresados de carreras profesionales de Universidad San Pedro. Tesis de maestria, Universidad San Pedro, Chimbote- Perú.

Universidad Nacional José Faustino Sánchez Carrión (2019), Portal de transparencia. Recuperado de http://app.unjfsc.edu.pe/transparencia/ZonaUsuarioResoluciones/Resoluciones.a $\underline{\mathrm{spx}}$ 